\title{
Raman and Computational Study on the Adsorption of Xanthine on Silver Nanocolloids
}

\author{
Francesco Muniz-Miranda, ${ }^{* \dagger, \|}$ Alfonso Pedone, ${ }^{\dagger}$ and Maurizio Muniz-Miranda ${ }^{\ddagger}, \S \odot$ \\ ${ }^{\dagger}$ Department of Chemical and Geological Sciences (DSCG), University of Modena and Reggio Emilia (UniMORE), Via Campi 103, \\ 41125 Modena, Italy \\ ${ }^{\ddagger}$ Department of Chemistry "Ugo Schiff”, University of Florence (UniFI), Via della Lastruccia 3, 50019 Sesto Fiorentino, Italy \\ ${ }^{\S}$ Institute of Complex Systems (CNR), Via Madonna del Piano 10, 50019 Sesto Fiorentino, Italy
}

Supporting Information

ABSTRACT: Xanthine is a nucleobase, deriving from adenine and guanine by deamination and oxidation processes, which may deposit in the human body causing diseases, similar to uric acid. Here, we have investigated the adsorption of xanthine on silver colloidal nanoparticles by means of surface-enhanced Raman scattering (SERS) with an exciting radiation in the near-infrared spectral region, where interference due to fluorescence does not occur, along with density functional theory calculations of molecule/metal model systems. By adopting a combined experimental and computational approach, we have identified the "marker" SERS bands of xanthine and the tautomer that preferentially binds the silver particles, as well as the molecular group involved in the interaction with metal. This investigation allows using the FT-SERS spectroscopy for biosensory and diagnostic purposes in body fluids, detecting abnormal levels of xanthine, and preventing metabolic diseases.
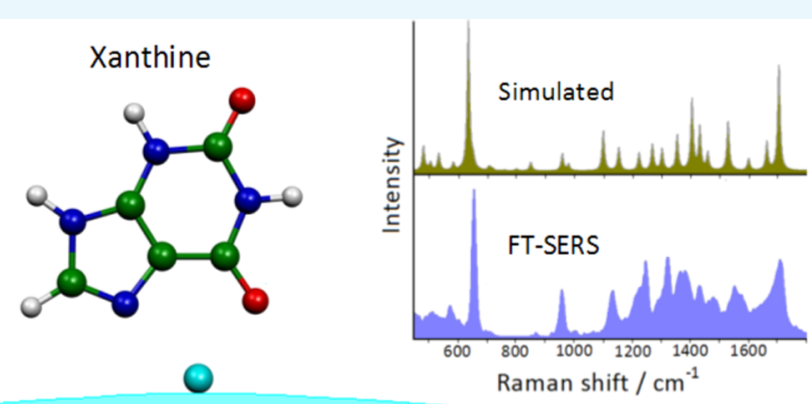

Ag nanoparticle

\section{INTRODUCTION}

The ability to recognize and quantitatively evaluate nucleotides, nucleosides, and their bases assumes great relevance in biomedical applications, ${ }^{1-3}$ mainly because it allows to easily monitor the degradation processes of nucleic acids present in body fluids and tissues. In fact, concentration changes of these components may pinpoint alterations in the physiological activity and presence of disease states. Xanthine is a purine base that is not commonly present in RNA or DNA chains yet derives from adenine and guanine by deamination and oxidation processes, as illustrated in Figure 1. Also, xanthine undergoes oxidation to uric acid, as shown in Figure 1, by the action of the xanthine oxidase enzyme.

As a consequence, a level of xanthine in the human body higher than normal increases the presence of uric acid, forming kidney stones with serious problems to the entire urinary tract. Moreover, xanthine, similar to uric acid, may deposit in the human body and cause diseases due to its scarce solubility in water. ${ }^{4,5}$ Hence, it is important to have a reliable and sensitive technique for the identification of xanthine in the human organism and prevent health-threatening conditions. In particular, the recent developments in high-performance liquid chromatography (HPLC) allow the simultaneous determination of purine metabolites as xanthine, hypoxanthine, and uric acid in the human plasma and serum. ${ }^{6}$ However, the possibility of evidencing the presence of xanthine in biological liquids through the surface-enhanced Raman scattering
(SERS) spectroscopy ${ }^{7,8}$ represents a valid alternative to the HPLC technique. SERS allows obtaining a trace detection of different molecules when the latter are adsorbed on nanostructured surfaces of noble metals as silver or gold. Actually, unlike the normal Raman scattering that has scarce sensitivity due to its small cross section, SERS ensures huge enhancements of the spectral signal of the adsorbed molecules, with factors generally up to $10^{6}-10^{7}$ with respect to the Raman response due to nonadsorbed ones but up to $10^{14}-10^{15}$ factors in single-molecule experiments. ${ }^{9,10}$ However, the validity of this spectroscopic technique is not only and not so much due to the sensitivity of the technique but (1) in the easy molecular recognition of the "markers" bands of the molecule and (2) in the simplicity of the investigation procedures that do not need any sample manipulation or separation procedure.

Here, we study the SERS response of xanthine adsorbed on silver colloidal nanoparticles, in view of analytical applications in biomedicine. For this purpose, we employed the FT-Raman instrumentation, with excitation in the near-infrared (NIR) spectral region. This excitation, despite the low intensity of the Raman response in the NIR region (owing to the $\lambda^{-4}$ dependence of Raman scattering intensity), is highly desirable for all of these applications requiring removal of any

Received: August 26, 2018

Accepted: October 8, 2018

Published: October 18, 2018 

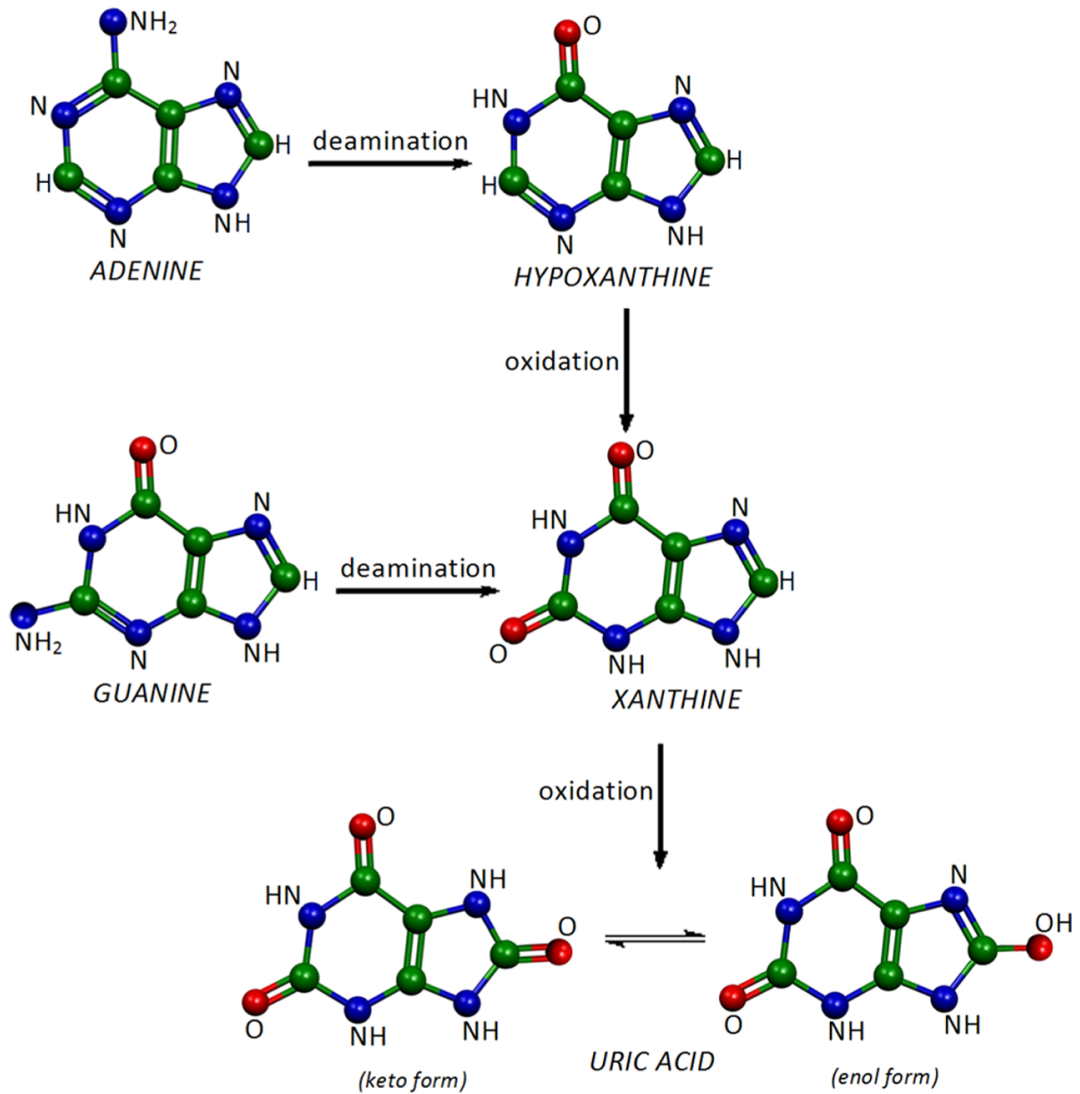

Figure 1. Formation of xanthine and uric acid from adenine and guanine.

interference of fluorescence from the Raman response. In fact, fluorescence emission can be due to impurities that, even in traces, add high levels of shot noise on the Raman bands, making their observation a difficult task, as often occurring in biological samples. ${ }^{11}$ Moreover, the NIR region being coincident with the so-called biological tissue transparency window, ${ }^{12}$ this could permit the use of the SERS spectroscopy to recognize the presence of xanthine both in vitro and in vivo.

The interpretation of the SERS data can be efficiently performed by means of density functional theory (DFT) calculations based on model systems constituted of molecules bound to adatoms or adclusters with a few metal atoms, which are able to simulate the active sites present on the surface of the colloidal nanoparticles. ${ }^{13-16}$ This method allows not only to reproduce accurately the observed SERS profiles, including band positions and relative intensities, but also to understand the type and strength of the molecule/metal interactions. Moreover, the DFT approach showed to be particularly useful in identifying the molecular species that chemically interact with metal. ${ }^{17-19}$ This information is essential to identify and quantify the presence of xanthine in a mixture of different compounds, as expected in body fluids, as well as to design analytical protocols to perform such analysis.

\section{RESULTS}

Structure and FT-Raman Spectra of Xanthine. As xanthine can exist as many possible tautomers, ${ }^{20}$ knowing the precise tautomers of this nucleobase is crucial to understand the mechanisms of the enzymes of which it is a substrate. However, the di-ketoforms, the $\mathrm{N}(9) \mathrm{H}$ and $\mathrm{N}(7) \mathrm{H}$ tautomers (see Figure 2), were considered by far the most probable with

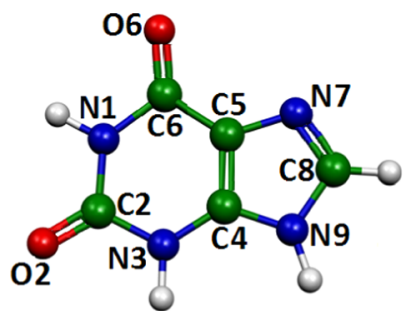

$\mathrm{N}(9) \mathrm{H}$ tautomer

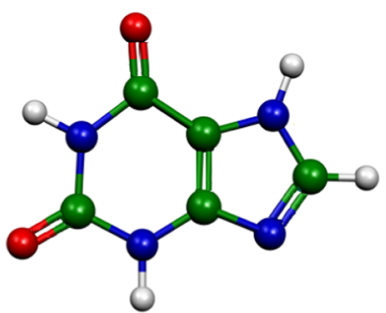

$\mathrm{N}(7) \mathrm{H}$ tautomer
Figure 2. Di-keto tautomeric forms of xanthine.

respect to those with enolic structures, on the basis of the energies estimated by AM1-PM3 study ${ }^{21}$ and DFT calculations; $^{20,22,23}$ these latter, moreover, indicated the $\mathrm{N}(7) \mathrm{H}$ tautomer as more stable than the $\mathrm{N}(9) \mathrm{H}$ tautomer. However, in acidic-neutral water solutions, where xanthine is not deprotonated, both tautomers are expected to be present. ${ }^{24}$

We have obtained FT-Raman spectra of xanthine in aqueous solutions at different $\mathrm{pH}$ values, as shown in Figure 3. It is important to show these spectra to observe possible similarities with the SERS spectrum of xanthine, which could suggest which molecular species is really adsorbed on silver. Moreover, the Raman spectra of xanthine in solid phase (see Figure 3), where the presence of the $\mathrm{N}(7) \mathrm{H}$ tautomer is expected, can provide information on the possible adsorption of this species on silver.

Sizeable Raman differences occur in aqueous solutions with respect to the Raman spectrum of the solid sample and also in solution by varying the $\mathrm{pH}$ values. At $\mathrm{pH}=6$ value, where xanthine, having $\mathrm{p} K_{\mathrm{a}}=7.60$, is predominantly present as 


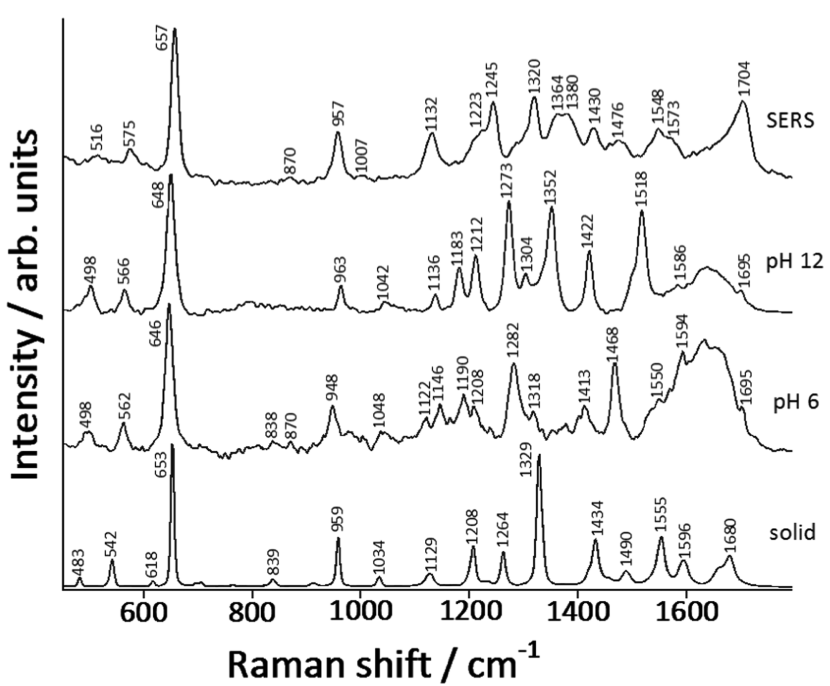

Figure 3. Normal Raman spectra of xanthine as a solid sample and dissolved in water solutions at different $\mathrm{pH}$ values, in comparison with the corresponding SERS spectrum in Ag colloid. Concentrations: $10^{-1}$ $\mathrm{M}$ (water, $\mathrm{pH} 12$ ), $10^{-3} \mathrm{M}$ (water, $\mathrm{pH}$ 6), and $10^{-6} \mathrm{M}$ (Ag colloid). Excitation: $1064 \mathrm{~nm}$.

neutral molecule, the Raman spectrum is quite weak, because neutral xanthine is scarcely soluble in water. At $\mathrm{pH}=12$, instead, the Raman spectrum of the deprotonated xanthine is quite intense, because high concentrations (around $10^{-1} \mathrm{M}$ ) can be obtained in water. However, the strongest band occurs in all spectra around $650 \mathrm{~cm}^{-1}$; this band, for which position and intensity do not significantly change by varying the physical state and the $\mathrm{pH}$ value in solution, can be confidently attributed to the breathing mode of the ring system of xanthine. The band observed at $1680 \mathrm{~cm}^{-1}$ in the solid Raman spectrum is due to $\mathrm{C}=\mathrm{O}$ stretching mode, with corresponding bands occurring around $1695 \mathrm{~cm}^{-1}$ in the aqueous solutions. The observed frequencies are reported in Table S1 of the Supporting Information; the solid Raman frequencies observed in the present study are similar to those previously reported in 2004 and $2005^{25,26}$ but differ from some bands reported in $2011,{ }^{27}$ in both positions and relative intensities. This evidence could be attributed to a lower purity degree of the sample examined in 2011.

Adsorption of Xanthine on Silver Colloidal Nanoparticles. The aim of this paper, devoted to the SERS detection of xanthine, is to use a metal colloidal suspension that exhibits these two characteristics: colloidal stability and SERS efficiency. Therefore, we chose to use a silver colloid obtained by reduction of silver ions with citrate, according to the well-tested procedure by Lee and Meisel, ${ }^{28}$ because it exhibits a marked stability in both absence and presence of organic ligand, thanks to the protection ensured by adsorbed citrate anions. This is particularly important for the identification and quantitative determination of xanthine, since colloidal aggregation processes that evolve in time could significantly alter the SERS response.

Several studies were performed to characterize the silver colloids obtained by reduction with citrate according to Lee and Meisel. ${ }^{28}$ It is important to point out that boiling the colloidal suspension caused silver nanoparticles to be monodispersed by the capping effect of the citrate anions, with maximum absorption of the plasmonic band around 420 $\mathrm{nm}{ }^{28}$ The resulting $\mathrm{pH}$ value was around $6.5 .^{29} \mathrm{In}$ our case, the $\mathrm{pH}$ is slightly lower $(\mathrm{pH} \sim 6)$, because we waited a few weeks after the colloid preparation before using the $\mathrm{Ag}$ suspension to obtain a better stabilization.

After addition of xanthine $\left(10^{-6} \mathrm{M}\right.$ concentration) to the citrate-reduced $\mathrm{Ag}$ colloid, the maximum of the plasmon band of the silver nanoparticles occurs at $425 \mathrm{~nm}$ wavelength, with a tail in the red-light region (see Figure 4). In this situation, no

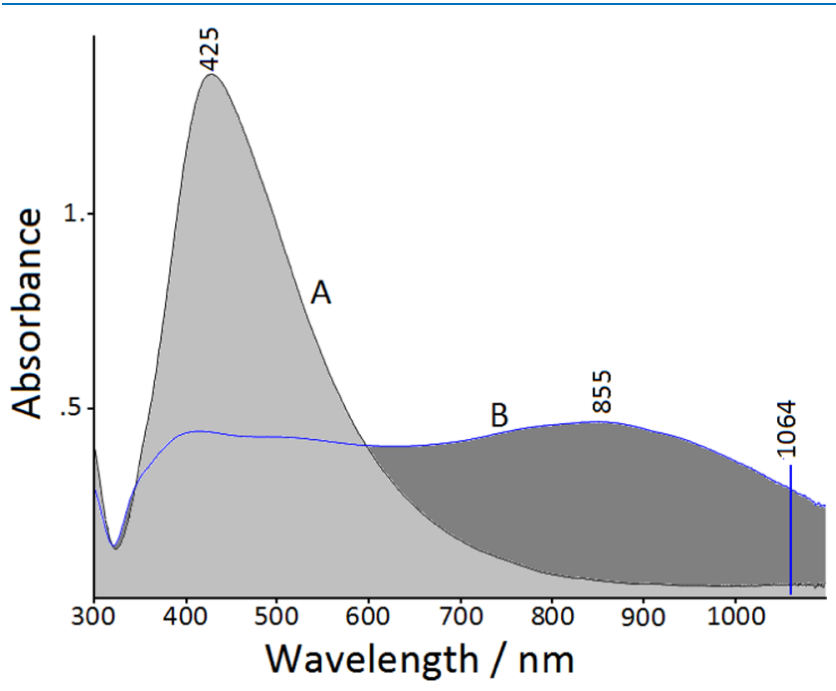

Figure 4. UV-visible extinction spectra of a silver colloid with $10^{-6}$ $\mathrm{M}$ xanthine, before (A) and after (B) addition of $10^{-3} \mathrm{M} \mathrm{NaCl}$. The $1064 \mathrm{~nm}$ laser line of the FT-Raman instrument is indicated.

FT-SERS signal can be detected by laser excitation at $1064 \mathrm{~nm}$, because the excitation wavelength does not match the plasmon band. However, by adding $\mathrm{NaCl}\left(10^{-3} \mathrm{M}\right.$ concentration), the plasmon band markedly moves to the infrared region, due to the aggregation of the silver particles promoted by the presence of co-adsorbed chloride anions. In this case, strong FT-SERS bands can be observed. The SERS activation of the silver colloids by excitation in the NIR region was previously ascertained and discussed by Kiefer and co-workers. ${ }^{30-32}$

FT-SERS Spectra of Xanthine in Silver Colloids. Figure 3 (upper panel) shows the FT-SERS spectrum of xanthine adsorbed on Ag colloid, which appears dominated by a very strong band at $657 \mathrm{~cm}^{-1}$, similar to what observed in the normal Raman spectra of xanthine in solutions and in solid phase. The SERS spectrum is quite different, in both band frequencies and intensities, from the very weak FT-SERS spectrum previously reported; ${ }^{33}$ the band positions, instead, appear similar to those obtained by Cotton and co-workers by visible excitation. ${ }^{34}$ At the $\mathrm{pH}$ value of the Ag colloid $(\sim \mathrm{pH}=$ 6 ), xanthine is expected to adsorb on silver as a neutral molecule, which is predominantly present in aqueous solution at this $\mathrm{pH}$ value. This, however, does not mean that the molecule in silver colloidal suspension at this $\mathrm{pH}$ value cannot bind to the metal as a deprotonated anion. In fact, when a molecule chemically interacts with silver colloidal nanoparticles, deprotonation reactions may occur by effect of the metal surface, although the deprotonated species is absolutely negligible at the $\mathrm{pH}$ value of the aqueous solution. In our case, however, the strong Raman bands of deprotonated xanthine observed at 1273,1352 , and $1518 \mathrm{~cm}^{-1}$ in the Raman spectrum of the alkaline solution (see Figure 3) do not find any correspondence in the SERS spectrum. This leads to exclude an adsorption of xanthine in anionic form. Moreover, 

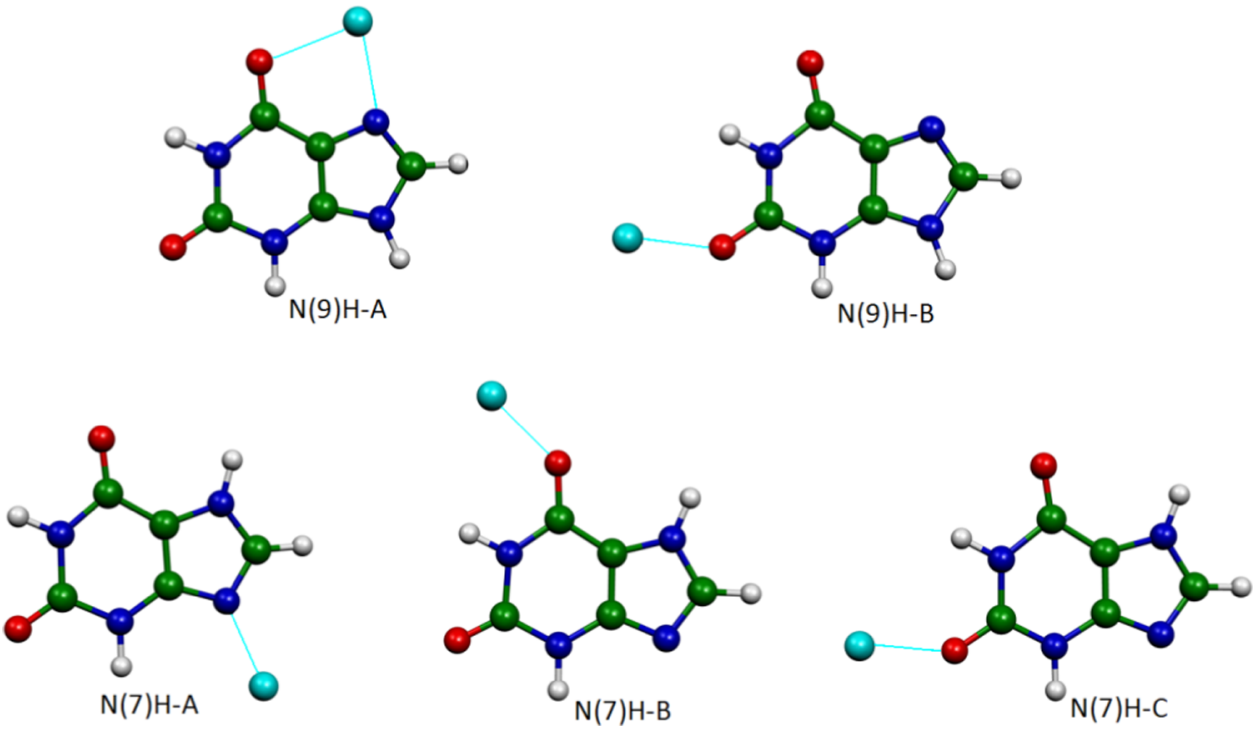

Figure 5. DFT-optimized (B3LYP/def2TZVPP) structures of the xanthine/silver complexes.

the SERS spectrum appear quite different also from the normal Raman spectrum of the solid sample (see Table S1 in the Supporting Information), where the molecule is present as $\mathrm{N}(7) \mathrm{H}$ tautomer, suggesting this latter is not involved in the interaction with metal. Spectral differences, however, occur in the SERS spectrum also with respect to the normal Raman spectrum of the aqueous solution at $\mathrm{pH}=6$, where the coexistence of both tautomers is expected. Besides marked frequency shifts observed in the $1200-1700 \mathrm{~cm}^{-1}$ spectral region, the highest-frequency band occurs in the SERS spectrum beyond $1700 \mathrm{~cm}^{-1}$, unlike in the normal Raman spectra. The FT-SERS spectrum of xanthine in Ag colloid is similar to that of guanine adsorbed on silver, ${ }^{35}$ especially by considering the dominant band around $650 \mathrm{~cm}^{-1}$ and the highfrequency band at about $1710 \mathrm{~cm}^{-1}$.

The spectral differences between SERS and Raman spectra of xanthine are attributable to the interaction with silver, which is to be interpreted as chemisorption, which significantly modifies the force constants of the molecule as well as their relative Raman intensities. For this reason, the DFT approach could provide important information on the adsorption of xanthine, concerning the preferential tautomer interacting with metal, the molecular site of interaction with the active sites of the Ag nanoparticles, and also the nature of the latter. We have adopted five different complexes of both tautomers of xanthine linked to silver nanoparticles through the nitrogen atoms with $\mathrm{sp}^{2}$ hybridization or the oxygen atoms of the carbonyl groups. The metal active sites are simply modeled as $\mathrm{Ag}^{+}$adatoms, as previously carried out for adsorbed adenine. ${ }^{14}$ The DFToptimized structures of these complexes are shown in Figure 5. The $\mathrm{N}(9) \mathrm{H}$ tautomer can link the silver adatom via $\mathrm{N} 7$ or $\mathrm{O} 10$, in the $\mathrm{N}(9) \mathrm{H}-\mathrm{A}$ or $\mathrm{N}(9) \mathrm{H}-\mathrm{B}$ complexes, respectively. In the first case, also the $\mathrm{O} 11$ atom of the $\mathrm{C}=\mathrm{O}$ group vicinal to $\mathrm{N} 7$ is involved in the binding with the metal surface. The tautomer $\mathrm{N}(7) \mathrm{H}$, instead, can link the silver adatom via $\mathrm{N} 9$, $\mathrm{O} 6$, or $\mathrm{O} 2$, in the $\mathrm{N}(7) \mathrm{H}-\mathrm{A}, \mathrm{N}(7) \mathrm{H}-\mathrm{B}$, or $\mathrm{N}(7) \mathrm{H}-\mathrm{C}$ complexes, respectively.

DFT Simulations of the SERS Spectrum of Xanthine in Silver Colloid. SERS enhancement is generally considered ${ }^{7,8}$ as the product of two contributions, one due to a long-range electromagnetic mechanism and another due to a short-range chemical mechanism. Regarding the first one, the molecules approaching a noble-metal nanoparticle undergo an electric field several orders of magnitude (at least four) larger than that at long distances from the surface, under the action of the electromagnetic radiation. The second contribution to the SERS enhancement, instead, depends on the change of the molecular polarizability due to the formation of chemical bonds with the active sites of the metal surface. Although the electromagnetic contribution plays a predominant role for the overall SERS enhancement, the chemical one is important to determine the SERS spectral pattern, concerning both spectral positions and relative intensities of the observed bands. Hence, for chemisorbed molecules, the profiles of the SERS spectra are essentially determined by the chemical interactions between the molecules and the active sites of the metal surface, called adatoms, constituted by one or a few atoms, as stated by Otto $^{36}$ and Aroca. ${ }^{37}$ The validity of the adatom approximation was widely verified by DFT calculations of many adsorbed molecules; in particular, the DFT-calculated Raman spectra of molecule/metal complexes with a single silver atom are quite often able to reproduce satisfactorily the corresponding SERS spectra, as shown also very recently. ${ }^{38-40}$ It is important to underline that the adatoms present on the surface of the silver colloidal nanoparticles can be effectively considered positively charged. ${ }^{12-14}$ In this regard, the adsorption of the negatively charged citrate ions on the silver colloidal surface plays an important role: the citrate anions not only perform a stabilizing action of the colloidal suspension but also promote the formation of positive charges on the silver surface.

For our DFT calculations on the complexes of xanthine linked to one Ag ion, we used the hybrid functional B3LYP in combination with two different basis sets: def2TZVPP and a mixed basis set $6-311 \mathrm{G}++(\mathrm{d}, \mathrm{p})$ for all atoms except silver, treated with Lanl2DZ. Table S2 (Supporting Information) reports the energies of the complexes calculated with the def2TVZPP basis set. The N(9)H-A complex, where the silver adatom is linked to the $\mathrm{N}(9) \mathrm{H}$ tautomer in a bidentate way, is by far the most stable, whereas the N(9)H-B complex exhibits marked instability. The complexes with the $\mathrm{N}(7) \mathrm{H}$ tautomer present similar energies. Moreover, the N(9)H-A complex provides the largest molecule $\rightarrow$ metal charge transfer (Table 
S2 in the Supporting Information), as obtained from the Hirshfeld partial charges, ${ }^{41}$ reported in Table S3 (Supporting Information) for the $\mathrm{N}(9) \mathrm{H}-\mathrm{A}$ complex.

As shown in Table 1, only the complex $\mathrm{N}(9) \mathrm{H}-\mathrm{A}$ is able to reproduce satisfactorily all observed SERS frequencies, in

Table 1. Observed and Calculated ${ }^{a}$ SERS Frequencies of Xanthine

\begin{tabular}{|c|c|c|c|c|c|}
\hline $\begin{array}{l}\text { FT-SERS } \\
\text { Ag colloid }\end{array}$ & $\begin{array}{c}\text { calcd } \\
\mathrm{N}(9) \mathrm{H}-\mathrm{A} \\
\text { complex }\end{array}$ & $\begin{array}{c}\text { calcd } \\
\mathrm{N}(9) \mathrm{H}-\mathrm{B} \\
\text { complex }\end{array}$ & $\begin{array}{c}\text { calcd } \\
\mathrm{N}(7) \mathrm{H}-\mathrm{A} \\
\text { complex }\end{array}$ & $\begin{array}{c}\text { calcd } \\
\mathrm{N}(7) \mathrm{H}-\mathrm{B} \\
\text { complex }\end{array}$ & $\begin{array}{c}\text { calcd } \\
\mathrm{N}(7) \mathrm{H}-\mathrm{C} \\
\text { complex }\end{array}$ \\
\hline 516 & 531 & 530 & 533 & 502 & 545 \\
\hline 575 & 582 & 539 & & 597 & 583 \\
\hline 657 & 634 & 623 & 627 & 656 & 634 \\
\hline 870 & 849 & 858 & 857 & 845 & 862 \\
\hline 957 & 956 & 950 & 961 & 956 & 965 \\
\hline 1132 & 1150 & & 1123 & 1126 & 1122 \\
\hline 1223 & 1221 & & 1214 & 1224 & 1206 \\
\hline 1245 & 1268 & 1278 & 1261 & 1248 & 1283 \\
\hline 1320 & 1303 & 1339 & 1305 & 1330 & 1313 \\
\hline 1364 & 1351 & 1365 & & & 1374 \\
\hline 1380 & 1404 & 1398 & & 1393 & 1387 \\
\hline 1430 & 1430 & & 1421 & 1454 & 1457 \\
\hline 1476 & 1458 & 1465 & 1458 & 1481 & 1476 \\
\hline 1551 & 1529 & 1521 & & & \\
\hline 1573 & 1598 & 1597 & 1593 & 1581 & 1574 \\
\hline 1704 & 1703 & & & & \\
\hline
\end{tabular}

particular, those occurring in the $1200-1550 \mathrm{~cm}^{-1}$ spectral region and that around $1700 \mathrm{~cm}^{-1}$. Quite similar results are obtained by using the mixed basis set, 6-311G++(d,p)/ Lanl2DZ. Regarding the higher-frequency bands, it can be observed that the stretching vibrations of the $\mathrm{C}=\mathrm{O}$ bonds that are not engaged in the interaction with metal are overestimated by using both def2TZVPP and the mixed basis set, as previously observed, ${ }^{42}$ because the interactions with the molecules of the water solvent are not taken into account in our calculation models. Actually, it was ascertained that the $\mathrm{C}=\mathrm{O}$ stretching frequencies are largely overestimated when the protic solvent is not considered in the DFT calculations. ${ }^{43-45}$

These computational results suggest that the preferential adsorption of xanthine on silver occurs with the $\mathrm{N}(9) \mathrm{H}$ tautomer, by involving both the N7 atom and the oxygen atom of the vicinal carbonyl group. This is confirmed by comparing the observed SERS spectrum with that calculated with all complexes, as shown in Figure 6 in the case of the def2TVZPP basis set. Very similar results, by considering band frequencies and intensities, are obtained with the mixed basis set, as shown in Figure S1 (Supporting Information). Only the $\mathrm{N}(9) \mathrm{H}-\mathrm{A}$ complex can simulate satisfactorily all experimental features, regarding both positions and relative intensities of the SERS bands. In particular, only this model is able to show the dominance of the SERS band around $650 \mathrm{~cm}^{-1}$, as well as the occurrence of the strong SERS band around $1700 \mathrm{~cm}^{-1}$.

Finally, Figure 7 shows the normal modes, calculated with the def2TZVPP basis set, corresponding to the prominent SERS bands, observed at $647,957,1245,1320$, and $1704 \mathrm{~cm}^{-1}$, which may be considered as "marker" bands of xanthine adsorbed on silver. All of these bands are assigned to in-plane vibrations; in particular, the dominant band at $657 \mathrm{~cm}^{-1}$ is

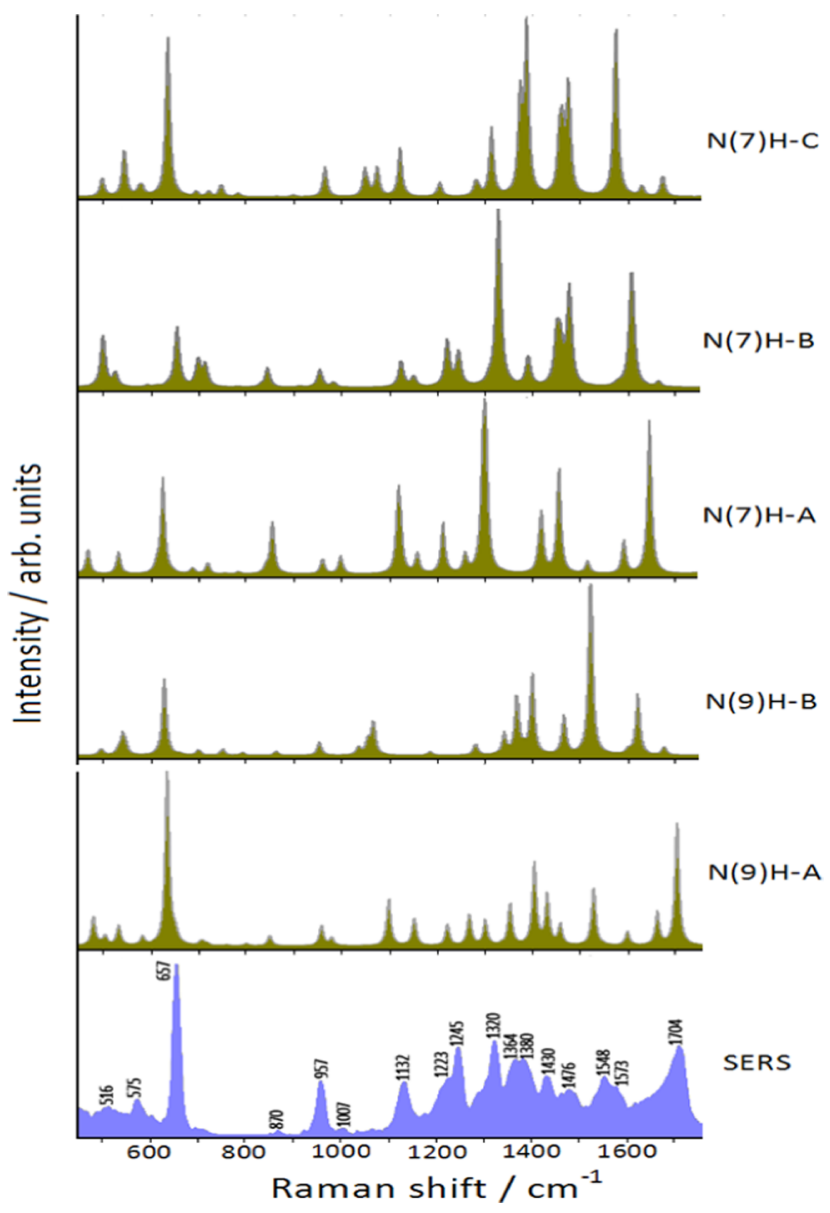

Figure 6. DFT-simulated (B3LYP/def2TZVPP) SERS spectra of the xanthine/silver complexes, compared with the observed SERS spectrum of xanthine adsorbed on Ag nanoparticles.

attributable to the ring breathing vibration, that at $957 \mathrm{~cm}^{-1}$ is attributable to deformation of the imidazolic ring, those at 1245 and $1320 \mathrm{~cm}^{-1}$ are attributable to ring deformations mixed with $\mathrm{N}-\mathrm{H}$ bending vibrations, whereas that at $1704 \mathrm{~cm}^{-1}$ is attributable to the stretching mode of the $\mathrm{C}=\mathrm{O}$ bond involved in the binding with silver.

\section{CONCLUDING REMARKS}

Xanthine is a purine nucleobase that is not present in DNA/ RNA chains but derives from the metabolic degradation of guanine and adenine, as described in Figure 1. Abnormal levels of xanthine and uric acid (oxidation product of xanthine) in the human body lead to the emergence of severe pathologies. This work has shown how it is possible to use the SERS spectroscopy in the early diagnosis of these diseases by determining the presence of xanthine in body fluids. For this aim, we have obtained a strong SERS activation in the NIR region, where fluorescence phenomena that interfere with the SERS signal do not usually occur. Moreover, the use of FTRaman spectroscopy can allow the SERS investigation both in vitro and in vivo, given that the laser exciting radiation at $\lambda=$ $1064 \mathrm{~nm}$ falls in the biological tissue transparency window. In the present case, the strong FT-SERS activation is due to both electromagnetic and chemical mechanisms: the first is due to the shift of the plasmon band toward the infrared region; the second one is due to the formation of charge-transfer complexes induced by the presence of chloride anions. ${ }^{46}$ 

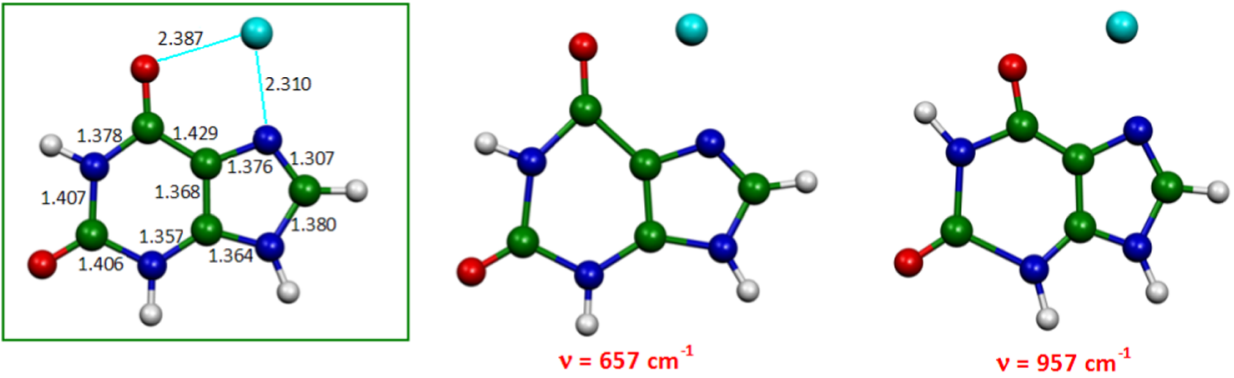

$v=957 \mathrm{~cm}^{-1}$
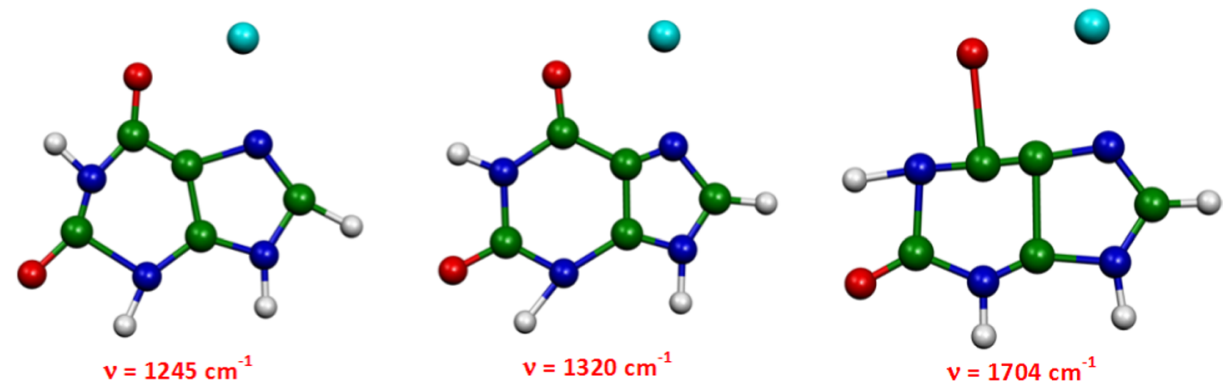

Figure 7. Optimized geometry of the $\mathrm{N}(9) \mathrm{H}-\mathrm{A}$ complex with distances in angstroms, along with the normal modes corresponding to the prominent SERS bands observed in the Ag colloid.

We have identified and assigned the marker bands in the SERS spectrum of xanthine to detect this nucleobase in the presence of other organic components. To do this, we have used the DFT computational approach, as the interpretation of the SERS spectrum of xanthine is complicated by the possible presence of two tautomers and of different complexes formed by molecule-metal interactions. In particular, the DFT simulations, based both on the frequencies and relative intensities of the calculated spectra and on the calculated energies of the different complexes, have allowed: (i) to recognize the tautomer $\mathrm{N}(9) \mathrm{H}$ as the molecular species linked to the silver nanoparticles, (ii) to identify the molecular site involved in the interaction with silver, that is, the N7 atom together with the vicinal carbonyl group, (iii) to highlight the interaction of xanthine with silver, that is, a strong chemisorption with a marked molecule $\rightarrow$ metal charge transfer, and (iiii) to evidence the nature of the active sites of the metallic surface, which are effectively modeled by positively charged silver atoms.

\section{EXPERIMENTAL SECTION}

Preparation of Silver Colloids. Stable Ag colloids were prepared in triple distilled water by reducing silver nitrate with sodium citrate at its boiling point, according to Lee and Meisel. ${ }^{28}$ Sodium citrate dehydrate (Sigma, purity $\geq 99.5 \%$ ) was added dropwise to $\mathrm{AgNO}_{3}$ (Aldrich, purity 99.9999\%) aqueous solution under vigorous stirring; the presence of citrate ensured long-term colloidal stability. Silver colloids, used some weeks after the preparation, had a $\mathrm{pH}$ value around 6. Xanthine (purity $\geq 99.5 \%$ ), supplied by Sigma as purified by recrystallization, was added to the $\mathrm{Ag}$ colloidal suspension in a $10^{-6} \mathrm{M}$ final concentration. To observe an FT-SERS signal of xanthine, $\mathrm{NaCl}$ was added to the $\mathrm{Ag}$ colloid in a $10^{-3} \mathrm{M}$ final concentration.

UV-Visible Absorption Measurements. Absorption spectra of the silver colloidal suspensions were observed in the $200-1100 \mathrm{~nm}$ region by means of a Cary 5 Varian spectrophotometer.
Raman Measurements. Raman spectra of xanthine as a solid sample, dissolved in acidic-neutral (around $\mathrm{pH}=6$ ) and alkaline (around $\mathrm{pH}=12$ ) aqueous solutions or adsorbed on Ag colloidal nanoparticles, were recorded with a Fourier transform (FT)-Raman spectrometer (Bruker Optics, Model MultiRam), equipped with a broad-range quartz beamsplitter, an air-cooled Nd:YAG laser excitation source (1064 nm), and a Ge diode detector cooled with liquid nitrogen. The instrument provided a spectral range of 3600-50 $\mathrm{cm}^{-1}$ (Stokes shift). The experiments were performed in a $180^{\circ}$ geometry, with $200 \mathrm{~mW}$ of laser power.

\section{COMPUTATIONAL DETAILS}

Calculations were performed with the Gaussian 09 software. ${ }^{47}$ We adopted the hybrid B3LYP exchange-correlation functional, ${ }^{48,49}$ along two different basis sets: $\operatorname{def} 2 \mathrm{TZVPP}{ }^{50-52}$ and a mixed basis set made up of $6-311++G(d, p)$ for nonmetal atoms and Lanl2 $\mathrm{DZ}^{53-58}$ for silver. Calculations consisted of structural optimizations of the tautomers of xanthine linked to metal adatoms, followed by vibrational frequency calculations. We checked that all vibrational frequencies were real and positive, thus belonging to geometries corresponding to true potential energy minima.

The calculated Raman activities $\left(A_{\mathrm{i}}\right)$ were converted to relative Raman intensities $\left(I_{\mathrm{i}}\right)$ using the following relationship, as reported in the literature ${ }^{59,60}$

$$
I_{\mathrm{i}}=\frac{f\left(\nu_{\mathrm{o}}-\nu_{\mathrm{i}}\right)^{4} A_{\mathrm{i}}}{\nu_{\mathrm{i}}\left(1-\mathrm{e}^{-h c \nu_{\mathrm{i}} / k T}\right)}
$$

where $\nu_{\mathrm{o}}$ is the exciting frequency (in $\mathrm{cm}^{-1}$ units); $\nu_{\mathrm{i}}$ is the vibrational frequency (in $\mathrm{cm}^{-1}$ units) of the $i_{\text {th }}$ normal mode; $h$, $c$, and $k$ are fundamental constants; and $f$ is a suitably chosen common normalization factor for all peak intensities. 


\section{ASSOCIATED CONTENT}

\section{S Supporting Information}

The Supporting Information is available free of charge on the ACS Publications website at DOI: 10.1021/acsomega.8b02174.

Observed Raman frequencies of xanthine; calculated energies and molecule $\rightarrow$ metal electronic charge transfers in silver complexes; Hirshfeld atomic charges; comparison between the simulated SERS spectra of the $\mathrm{N}(9) \mathrm{H}-\mathrm{A}$ complex, obtained with the $6-311 \mathrm{G}++(\mathrm{d}, \mathrm{p}) /$ Lanl2DZ and def2TZVPP basis sets (PDF)

\section{AUTHOR INFORMATION}

\section{Corresponding Author}

*E-mail: francesco.munizmiranda@ugent.be, f. munizmiranda@gmail.com.

\section{ORCID $\odot$}

Alfonso Pedone: 0000-0003-3772-7222

Maurizio Muniz-Miranda: 0000-0001-9457-6833

\section{Present Address}

"Post-doctoral fellow at the Department of Physics and Astronomy, Center for Molecular Modeling (CMM), Ghent University (UGent), Technologiepark 903, 9052 Zwijnaarde, Belgium (F.M.M.).

\section{Notes}

The authors declare no competing financial interest.

\section{ACKNOWLEDGMENTS}

FM-M's post-doctoral fellowship at UniMORE in Prof. Dr. Alfonso Pedone's group during the 2013-2017 period was supported by the Italian "Ministero dell'Istruzione, dell'Università e della Ricerca" (MIUR) through the "Futuro in Ricerca" (FIRB) Grant RBFR1248UI 002 entitled "Novel Multiscale Theoretical/Computational Strategies for the Design of Photo and Thermo responsive Hybrid OrganicInorganic Components for Nanoelectronic Circuits”.

\section{REFERENCES}

(1) Eichhorn, G. L. The effect of metal ions on the structure and function of nucleic acids. In Advances in Inorganic Biochemistry, Eichhorn, Gunther L., Marzilli, Luigi G., Eds.; Elsevier: New York, 1981, Vol. 3, pp. 1-46, ISBN-10: 0444006370.

(2) Azam, S.; Hadi, N.; Khan, N. U.; Hadi, S. M. Antioxidant and prooxidant properties of caffeine, theobromine and xanthine. Med. Sci. Monit. 2003, 9, BR325-330 PMID: 12960921.

(3) Blackburn, G. M. Nucleic Acids in Chemistry and Biology, 3rd ed.; RSC Pub.: Cambridge, 2006.

(4) Königsberger, E.; Wang, Z.; Seidel, J.; Wolf, G. Solubility and dissolution enthalpy of xanthine. J. Chem. Thermodyn. 2001, 33, 1-9.

(5) Ichida, K.; Amaya, Y.; Kamatani, N.; Nishino, T.; Hosoya, T.; Sakai, O. Identification of two mutations in human xanthine dehydrogenase gene responsible for classical type I xanthinuria. J. Clin. Invest. 1997, 99, 2391-2397.

(6) Pleskačová, A.; Brejcha, S.; Pácal, L.; Kaňková, K.; Tomandl, J. Simultaneous Determination of Uric Acid, Xanthine and Hypoxanthine in Human Plasma and Serum by HPLC-UV: Uric Acid Metabolism Tracking. Chromatographia 2017, 80, 529-536.

(7) Schlücker, S. Surface Enhanced Raman Spectroscopy: Analytical, Biophysical and Life Science Applications; Wiley-VCH: Weinheim, Germany, 2011.

(8) Procházka, M. Surface-Enhanced Raman Spectroscopy, Bioanalytical, Biomolecular and Medical Applications; Springer: Switzerland, 2016.
(9) Kneipp, K.; Wang, Y.; Kneipp, H.; Perelman, L. T.; Itzkan, I.; Dasari, R. R.; Feld, M. S. Single Molecule Detection Using SurfaceEnhanced Raman Scattering (SERS). Phys. Rev. Lett. 1997, 78, 16671670.

(10) Nie, S.; Emory, S. R. Probing Single Molecules and Single Nanoparticles by Surface-Enhanced Raman Scattering. Science 1997, 275, 1102-1106.

(11) Chase, D. B. Fourier transform Raman spectroscopy. J. Am. Chem. Soc. 1986, 108, 7485-7488.

(12) Smith, A. M.; Mancini, M. C.; Nie, S. Bioimaging: Second window for in vivo imaging. Nat. Nanotechnol. 2009, 4, 710-711.

(13) Owen, A. R.; Golden, J. W.; Price, A. S.; Henry, W. A.; Barker, W. R.; Perry, D. A. Surface-Enhanced Vibrational Spectroscopy and Density Functional Theory Study of Isoniazid Layers Adsorbed on Silver Nanostructures. J. Phys. Chem. C 2014, 118, 28959-28969.

(14) Pagliai, M.; Caporali, S.; Muniz-Miranda, M.; Pratesi, G.; Schettino, V. SERS, XPS, and DFT Study of Adenine Adsorption on Silver and Gold Surfaces. J. Phys. Chem. Lett. 2012, 3, 242-245.

(15) Muniz-Miranda, M.; Pagliai, M. Positively Charged Active Sites for the Adsorption of Five-Membered Heterocycles on Silver Colloids. J. Phys. Chem. C 2013, 117, 2328-2333.

(16) Muniz-Miranda, M.; Muniz-Miranda, F.; Pedone, A. Raman and DFT study of methimazole chemisorbed on gold colloidal nanoparticles. Phys. Chem. Chem. Phys. 2016, 18, 5974-5980.

(17) Maiti, N.; Thomas, S.; Debnath, A.; Kapoor, S. Raman and XPS study on the interaction of taurine with silver nanoparticles. RSC Adv. 2016, 6, 56406-56411.

(18) Dhayagude, A. C.; Maiti, N.; Debnath, A. K.; Joshi, S. S.; Kapoor, S. Metal nanoparticle catalyzed charge rearrangement in selenourea probed by surface-enhanced Raman scattering. RSC Adv. 2016, 6, 17405-17414.

(19) Muniz-Miranda, M.; Muniz-Miranda, F.; Caporali, S. SERS and DFT study of copper surfaces coated with corrosion inhibitor. Beilstein J. Nanotechnol. 2014, 5, 2489-2497.

(20) Polat, T.; Yildırım, G. Investigation of solvent polarity effect on molecular structure and vibrational spectrum of xanthine with the aid of quantum chemical computations. Spectrochim. Acta, Part A 2014, $123,98-109$.

(21) Civcir, P. Ü. AM1 and PM3 study of tautomerism of xanthine in the gas and aqueous phases. J. Mol. Struct.: THEOCHEM 2001, $545,7-15$.

(22) Kim, J. H.; Odutola, J. A.; Popham, J.; Jones, L.; von Laven, S. Tautomeric Energetics of Xanthine Oxidase Substrates: Xanthine, 2Oxo-6-methylpurine and Lumazine. J. Inorg. Biochem. 2001, 84, 145150

(23) Gobre, V. V.; Pinjari, R. V.; Gejji, S. P. Structure and Normal Vibrations in Xanthine and its Methyl derivatives from First Principle Calculations. J. Mol. Struct.: THEOCHEM 2010, 960, 86-92.

(24) Kulikowska, E.; Kierdaszuk, B.; Shugar, D. Xanthine, xanthosine and its nucleotides: solution structures of neutral and ionic forms, and relevance to substrate properties in various enzyme systems and metabolic pathways. Acta Biochim. Pol. 2004, 51, 493-531 DOI: 035001493 .

(25) Krishnakumar, M.; Arivazhagan, M. Vibrational and normal coordinate analysis of xanthine and hypoxanthine. Indian J. Pure Appl. Phys. 2004, 42, 411-418 IPC Code: GO1J 3/44 .

(26) Gunasekaran, S.; Sankari, G.; Ponnusamy, S. Vibrational spectral investigation on xanthine and its derivatives-theophylline, caffeine and theobromine. Spectrochim. Acta, Part A 2005, 61, 117127.

(27) Arivazhagan, M.; Jeyavijayan, S. FTIR and FT-Raman spectra, assignments, ab initio HF and DFT analysis of xanthine. Spectrochim. Acta, Part A 2011, 79, 161-168.

(28) Lee, P. C.; Meisel, D. Adsorption and surface-enhanced Raman of dyes on silver and gold sols. J. Phys. Chem. 1982, 86, 3391-3395.

(29) Cañamares, M. V.; Garcia-Ramos, J. V.; Gómez-Varga, J. D.; Domingo, C.; Sanchez-Cortes, S. Comparative Study of the Morphology, Aggregation, Adherence to Glass, and Surface-Enhanced Raman Scattering Activity of Silver Nanoparticles Prepared by 
Chemical Reduction of $\mathrm{Ag}^{+}$Using Citrate and Hydroxylamine. Langmuir 2005, 21, 8546-8553.

(30) Liang, E. J.; Engert, C.; Kiefer, W. Surface-enhanced Raman scattering of pyridine in silver colloids excited in the near-infrared region. J. Raman Spectrosc. 1993, 24, 775-779.

(31) Liang, E. J.; Kiefer, W. Chemical Effect of SERS with NearInfrared Excitation. J. Raman Spectrosc. 1996, 27, 879-885.

(32) Liang, E. J.; Ye, X. L.; Kiefer, W. Surface-Enhanced Raman Spectroscopy of Crystal Violet in the Presence of Halide and Halate Ions with Near-Infrared Wavelength Excitation. J. Phys. Chem. A 1997, 101, 7330-7335.

(33) Ranc, V.; Hruzikova, J.; Maitner, K.; Prucek, R.; Milde, D.; Kvitek, L. Quantification of purine basis in their mixtures at femtomolar concentration levels using FT-SERS. J. Raman Spectrosc. 2012, 43, 971-976.

(34) Sheng, R.; Ni, F.; Cotton, T. M. Determination of purine bases by reversed-phase high-performance liquid chromatography using real-time surface-enhanced Raman spectroscopy. Anal. Chem. 1991, 63, 437-442.

(35) Giese, B.; McNaughton, D. Density functional theoretical (DFT) and surface-enhanced Raman spectroscopic study of guanine and its alkylated derivatives. Phys. Chem. Chem. Phys. 2002, 4, 51715182.

(36) Otto, A. The 'chemical' (electronic) contribution to surfaceenhanced Raman scattering. J. Raman Spectrosc. 2005, 36, 497-509.

(37) Aroca, R. Surface-Enhanced Vibrational Spectroscopy; Wiley: New York, 2006.

(38) Wrzosek, B.; Cukras, J.; Dobrowolski, M. A.; Bukowska, J. Real Chemical States of the 3-Sulfur Derivative of 1,2,4-Triazole in Different Conditions: Complex Experimental and Theoretical Studies. J. Phys. Chem. C 2017, 121, 9282-9295.

(39) Harroun, S. G.; Zhang, Y.; Chen, T.-H.; Ku, C.-R.; Chang, H.T. Biomarkers of cigarette smoking and DNA methylating agents: Raman, SERS and DFT study of 3-methyladenine and 7methyladenine. Spectrochim. Acta, Part A 2017, 176, 1-7.

(40) Al-Shalalfeh, M. M.; Saleh, T. A.; Al-Saadi, A. A. Silver colloid and film substrates in surface-enhanced Raman scattering for 2thiouracil detection. RSC Adv. 2016, 6, 75282-75292.

(41) Saha, S.; Roy, R. K.; Ayers, P. W. Are the Hirshfeld and Mulliken population analysis schemes consistent with chemical intuition? Int. J. Quantum Chem. 2009, 109, 1790-1806.

(42) Gogia, S.; Jain, A.; Puranik, M. Structures, Ionization Equilibria, and Tautomerism of 6-Oxopurines in Solution. J. Phys. Chem. B 2009, 113, 15101-15118.

(43) Pagliai, M.; Muniz-Miranda, F.; Cardini, G.; Righini, R.; Schettino, V. Hydrogen bond dynamics of methyl acetate in methanol. J. Phys. Chem. Lett. 2010, 1, 2951-2955.

(44) Pagliai, M.; Muniz-Miranda, F.; Cardini, G.; Righini, R.; Schettino, V. Spectroscopic properties with a combined approach of $\mathrm{ab}$ initio molecular dynamics and wavelet analysis. J. Mol. Struct. 2011, 993, 438-442.

(45) Muniz-Miranda, F. Modelling of Spectroscopic and Structural Properties Using Molecular Dynamics; Firenze University Press (FUP): Italy, 2014 .

(46) Liu, Y.; Lu, Z.; Zhu, H.; Hasi, W. Characterization of a Chloride-Activated Surface Complex and Corresponding Enhancement Mechanism by SERS Saturation Effect. J. Phys. Chem. C 2017, 121, 950-957.

(47) Frisch, M. J.; Trucks, G. W.; Schlegel, H. B.; et al. Gaussian 09, revision D.01; Gaussian, Inc.: Wallingford, 2009.

(48) Becke, A. D. Density-functional thermochemistry. III. The role of exact exchange. J. Chem. Phys. 1993, 98, 5648-5652.

(49) Lee, C.; Yang, W.; Parr, R. G. Development of the ColleSalvetti correlation-energy formula into a functional of the electron density. Phys. Rev. B 1988, 37, 785-789.

(50) Weigend, F.; Ahlrichs, R. Balanced basis sets of split valence, triple zeta valence and quadruple zeta valence quality for $\mathrm{H}$ to $\mathrm{Rn}$ : Design and assessment of accuracy. Phys. Chem. Chem. Phys. 2005, 7, 3297-3305.
(51) Vosko, S. H.; Wilk, L.; Nusair, M. Accurate spin-dependent electron liquid correlation energies for local spin density calculations: a critical analysis. Can. J. Phys. 1980, 58, 1200-1211.

(52) Stephens, P. J.; Devlin, F. J.; Chabalowski, C. F.; Frisch, M. J. $\mathrm{Ab}$ Initio Calculation of Vibrational Absorption and Circular Dichroism Spectra Using Density Functional Force Fields. J. Phys. Chem. 1994, 98, 11623-11627.

(53) Hay, P. J.; Wadt, W. R. Ab initio effective core potentials for molecular calculations. Potentials for the transition metal atoms Sc to Hg. J. Chem. Phys. 1985, 82, 270-283.

(54) Wadt, W. R.; Hay, P. J. Ab initio effective core potentials for molecular calculations. Potentials for main group elements $\mathrm{Na}$ to $\mathrm{Bi} . J$. Chem. Phys. 1985, 82, 284-298.

(55) Hay, P. J.; Wadt, W. R. Ab initio effective core potentials for molecular calculations. Potentials for $\mathrm{K}$ to Au including the outermost core orbitals. J. Chem. Phys. 1985, 82, 299-310.

(56) Pagliai, M.; Muniz-Miranda, F.; Schettino, V.; Muniz-Miranda, M. Competitive Solvation and Chemisorption in Silver Colloidal Suspensions. Prog. Colloid Polym. Sci. 2011, 139, 39-44.

(57) Muniz-Miranda, M.; Pergolese, B.; Muniz-Miranda, F.; Caporali, S. SERS effect from Pd surfaces coated with thin films of Ag colloidal nanoparticles. J. Alloys Compd. 2014, 615, S357-S360.

(58) Gellini, C.; Deepak, F. L.; Muniz-Miranda, M.; Caporali, S.; Muniz-Miranda, F.; Pedone, A.; Innocenti, C.; Sangregorio, C. Magneto-Plasmonic Colloidal Nanoparticles Obtained by Laser Ablation of Nickel and Silver Targets in Water. J. Phys. Chem. C 2017, 121, 3597-3606.

(59) Keresztury, G. In Handbook of Vibrational Spectroscopy; Chalmers, J. M., Griffiths, P. R., Eds.; Wiley \& Sons: Chichester, UK, 2002; vol. 1; pp. 71-87.

(60) Krishnakumar, V.; Keresztury, G.; Sundius, T.; Seshadri, S. Density functional theory study of vibrational spectra and assignment of fundamental vibrational modes of 1-methyl-4-piperidone. Spectrochim. Acta, Part A 2007, 68, 845-850. 